Estudios Públicos 165 (2022), I39- I49

DOI: https://doi.org/|0.38178/07/83089/|31921 I009

Artículo

\title{
John Rawls y el problema de la desigualdad dentro y más allá de las fronteras
}

\author{
Isabel Turégano \\ Universidad de Castilla-La Mancha, España
}

RESUMEN: En la teoría de la justicia de John Rawls, la preocupación por la igualdad es central como imperativo de justificación recíproca de la estructura institucional básica. Esta propuesta sigue siendo adecuada para enfrentar el problema de la desigualdad en nuestros días. Su obra central, que ahora cumple 50 años, sigue proporcionándonos instrumentos para comprender las asimetrías de riqueza como asimetrías de poder. Algunas de las debilidades, no obstante, provienen de su concepción ambigua de esa estructura básica y de la idealización de su modelo de justicia, las que pueden distorsionar el modo de abordar la pluralidad de formas de desventaja que asolan los contextos no ideales. En todo caso, el compromiso igualitario de Rawls se vio esencialmente comprometido en su propuesta de un Derecho de Gentes que, comparado con la versión de la justicia que defendió para el marco estatal, es muy modesta. La supuesta coherencia de los fundamentos relacionales de su teoría de la justicia estatal e internacional no puede ser obstáculo para una teoría más exigente de la justicia global pensada desde algunas de las ideas centrales que Rawls nos legó.

PalABRAS Clave: Rawls, desigualdad, principio de diferencia, igualdad de oportunidades, justicia global

RECIBIDO: octubre 2021 / ACEPTADO: diciembre 2021

IsABel TuRÉGAno es Licenciada en Derecho por la Universidad de Castilla-La Mancha (UCLM), España. Profesora Titular de Filosofía del Derecho en la Facultad de Ciencias Sociales, UCLM. Dirección: Avda. de los Alfares 44, 16071, Cuenca, España. Email: isabel.turegano@uclm.es.

Este trabajo se ha elaborado en el marco del proyecto financiado por MCIN/AEI y FEDER (DOI: /10.13039/501100011033), 'Fronteras, democracia y justicia global. Argumentos filosóficos en torno a la emergencia de un espacio cosmopolita' (PGC2018-093656-B-I00). 


\title{
John Rawls and the Problem of Inequality within and beyond Borders
}

\begin{abstract}
In John Rawls's theory of justice, concern for equality is central as an imperative of reciprocal justification of the basic institutional structure. This proposal is still adequate to face the problem of inequality today. His central work, 50 years after its first edition, still provides us with tools to understand wealth asymmetries as power asymmetries. Some of the weaknesses, however, come from its ambiguous conception of that basic structure and from the idealization of his model of justice that can distort the way of dealing with the plurality of forms of disadvantage that plague non-ideal contexts. In any case, Rawls's egalitarian commitment was essentially compromised in his proposal for a Law of Peoples which, compared to the version of justice that he defended for the state framework, is very modest. The supposed coherence of the relational foundations of his theory of state and international justice cannot be an obstacle to a more demanding theory of global justice thought from some of the central ideas Rawls left us.
\end{abstract}

KEYWORDS: Rawls, inequality, difference principle, equality of opportunity, global justice

Received: October 2021 / Accepted: December 2021

a

a Teoría de la justicia de John Rawls (1995) incorpora una propuesta de justicia social sobre la que ha girado en gran parte el debate de estos últimos 50 años. Su pretensión es ofrecer un modelo que haga compatibles los valores de libertad e igualdad. Rawls escribe esta obra en el contexto de la guerra de Vietnam, persuadido de que en su sociedad la riqueza estaba mal distribuida y de que esto se traducía en una desigual influencia política y en un reparto desequilibrado de cargas. El sentido de su proyecto era construir una utopía realista de una vida colectiva valiosa, una sociedad ideal que se pudiera alcanzar a partir de las circunstancias de nuestro mundo y de nuestra naturaleza humana (Pogge 2007).

La aspiración a superar la desigualdad que está en la base de la propuesta abstracta de Rawls es un viejo problema que sigue siendo una amenaza central para las sociedades democráticas. Y sigue existiendo poco consenso acerca de aquello que hace insostenibles a las sociedades altamente desiguales y que merece ser atendido desde la política y el derecho. Las ideas de Rawls han marcado la línea de toda una serie de aportaciones esenciales de las últimas décadas en las que la desigualdad 
se considera objeto central de preocupación teórica y práctica porque socava la igual participación en sociedades conformadas para satisfacer cooperativamente las necesidades de todos. Su legado se inscribe, así, en la línea de un igualitarismo relacional o democrático que aspira a que todos los miembros de una comunidad se relacionen con los otros como iguales, esto es, una concepción de la justicia como esquema equitativo de cooperación social que sigue un imperativo de justificación recíproca entre iguales morales y políticos (Forst 2020).

A continuación me voy a referir a los elementos centrales de la propuesta igualitaria de John Rawls desde la perspectiva del contraste entre el modelo maximalista asociativo que propone para el nivel intraestatal y el modelo mínimo internacional. En primer lugar me refiero al principio de diferencia y al modelo igualitario para el ámbito estatal que se construye sobre él; en segundo lugar, al principio de igualdad de oportunidades y al modo en que condiciona ese modelo igualitario; y, por último, a la que considero la principal deficiencia de la apuesta de John Rawls por los más desaventajados: su restrictiva opción por un modelo internacionalista de justicia más allá de los Estados. Otras perspectivas de análisis crítico de su igualitarismo serían del mismo modo relevantes, pero exceden los límites de mi contribución a este simposio.

\section{El principio de diferencia}

La justicia para Rawls es una virtud de las instituciones. Su finalidad es determinar cómo han de regularse las instituciones de la estructura básica para que pueda mantenerse como un sistema eficiente y equitativo de cooperación social. En su esquema, el principio de libertad es equilibrado con los principios de igualdad justa de oportunidades y de diferencia. Conforme al segundo de ellos, la justicia social no aparece a priori centrada en la igualdad material de ingresos entre todos los miembros de la sociedad, sino que busca mejorar la situación de los más desfavorecidos. No es solo que las desigualdades económicas y sociales contribuyan al bien general o al beneficio de todos, sino específicamente al beneficio de los miembros menos aventajados de la sociedad. Para identificar al grupo de los menos aventajados, Rawls recurre a la idea de bienes primarios, como medios necesarios para que los ciudadanos puedan desarrollarse adecuadamente y ejercer sus facultades morales. 
Las diferencias a las que se refiere el principio rawlsiano son diferencias en las expectativas razonables de los ciudadanos respecto de los bienes primarios, siendo los menos aventajados aquellos que tienen las expectativas más bajas en relación con los ingresos (Rawls 2004, 90-94).

Esta restricción de la interpretación de la idea de los menos aventajados a los ingresos ha sido uno de los aspectos más discutidos de la teoría rawlsiana. Y es, quizás, uno de los temas más vivos en el debate actual sobre la desigualdad. La cuestión de si la discusión debe girar esencialmente en torno al asunto económico o si ha de orientarse al reconocimiento de las diferencias ha dominado gran parte de la literatura de los últimos años e hizo que el problema de la desigualdad económica pasara a un segundo plano. Pero la crisis económica de la segunda década del siglo XXI ha rescatado la centralidad de la desigualdad económica y su contradicción con la igualdad democrática, especialmente desde la publicación de Capital e ideología de Thomas Piketty (2019). Tanto para Rawls como para Piketty, las asimetrías de riqueza son también asimetrías de poder o, en palabras de Rawls, la libertad y la igualdad son dos caras del ideal democrático (Vallespín 2015).

La idea de igualdad que deriva del principio de diferencia va más allá de un principio distributivo que justifique transferencias e incluye la idea de reciprocidad. Aunque la desigualdad de ingresos pueda no ser injusta bajo ciertas condiciones, los menos aventajados pueden verse a sí mismos como miembros plenos en el mundo social solo si reconocen que las mayores ventajas de otros redundan en su propio bien (Rawls 2004, 176). El esquema rawlsiano no admitiría una interpretación libertaria conforme a la que la recompensa de los talentos individuales puede justificarse por el hecho de que aumente el nivel de beneficio social para todos, versión que podría considerarse reforzada por la noción de mínimo social que él incorpora (Rawls 1995, 258-259, 265-267, 292-293). El propio Rawls reinterpreta esta noción conforme a la idea de reciprocidad, considerando que "el concepto del mínimo adecuado no viene dado por las necesidades básicas de la naturaleza humana en sentido psicológico (o biológico) al margen de todo mundo social particular" (Rawls 2004, 179).

Cabe, pues, una interpretación fuertemente igualitaria del principio de diferencia que admita las diferencias solo cuando sea necesario para mejorar la posición de quienes están peor en términos de bienes prima- 
rios. Esta versión maximalista, además, es más igualitaria en el sentido de que, conforme a ella, el mejor esquema es el que atenúa las diferencias entre grupos en desigual posición, tal y como exigen el principio de ventaja mutua y el de igualdad democrática (Ribotta 2021, 287). Y esta otra interpretación es más coherente con la remisión que hace Rawls a esos otros valores que subyacen a la justicia como equidad, reciprocidad y fraternidad. Esta última la considera específicamente asociada con el principio de diferencia e implica incorporar un principio dirigido a los individuos conforme al que no han de querer mayores ventajas si no son para el beneficio de los peor situados (Rawls 1995, 107-108).

La propuesta igualitaria de Rawls supone una reforma del capitalismo. Aunque el principio de diferencia sirve para justificar la desigualdad y han sido muchos los que han visto en sus tesis el sustrato de un capitalismo de Estado de Bienestar, los últimos escritos muestran a un teórico 'poco amistoso con el capitalismo' y convencido de que la mejor traducción de su modelo de justicia como equidad es un socialismo democrático (Edmundson 2017). En esa línea, Thomas Piketty (2019, 1146-1147) ha sostenido sobre bases rawlsianas que el capitalismo tiene una tendencia inherente a crear altos niveles de desigualdad de ingresos y riqueza. Pero asumir esta propuesta supone un desafío central a la globalización económica y a los modelos restringidos de democracia que la acompañan, desafío al que, sin embargo, no hace frente John Rawls. Una síntesis entre el esquema de justicia de Rawls y la propuesta de Piketty de fiscalidad progresiva global y democracia transnacional generaría una poderosa crítica al modelo de capitalismo del Estado de Bienestar (Vallier 2019).

\section{Igualdad de oportunidades y estructura básica}

Al principio de diferencia precede, en el segundo principio de justicia rawlsiano, la igualdad de oportunidades. Rawls distingue su principio de equitativa igualdad de oportunidades de una versión más débil del mismo que denomina 'puestos abiertos a las capacidades', que exige que las posiciones ventajosas se abran a todos sobre una base competitiva en función de criterios imparciales de mérito (Rawls 1995, 88). En su propuesta descarta que el mérito sea un criterio distributivo asumible por los criterios de justicia (Rawls 1995, 287-291). La justa igualdad de oportunidades supone que la estructura básica debe ser organizada de 
modo que se disminuya la influencia moralmente injusta de factores arbitrarios. "La distribución natural", nos dice, "no es ni justa ni injusta [...] Estos son hechos meramente naturales. Lo que puede ser justo o injusto es el modo en que las instituciones actúan con respecto a estos hechos" (Rawls 1995, 104). Es la estructura básica la que no debe incorporar la arbitrariedad natural ni social.

Sin embargo, la idea de estructura básica de Rawls resulta insuficiente para identificar el complejo de procesos y estructuras que condicionan desigualitariamente la formación y realización de las preferencias y expectativas de los individuos. El propio Rawls considera que su concepto de estructura básica, integrada por las principales instituciones políticas, económicas y sociales que tienen efectos profundos sobre las vidas de las personas y distribuyen derechos y deberes, cargas y ventajas, es algo vago (Rawls 1995, 22). Gerald Cohen $(1997,18)$ ya se refirió a la "fatal ambigüedad" en la especificación que hace Rawls de la estructura básica.

La idea de igualdad de oportunidades basada en la competición voluntaria obvia las formas de estratificación social que condicionan el modo en que se definen las cualificaciones para las posiciones sociales a ocupar y el tipo de sujetos que pueden y desean acceder a ellas (Arneson 1999). De este modo, hace responsable al individuo de su situación y oculta la desigualdad real, legitimando y perpetuando el privilegio de algunos bajo la apariencia del mérito. Habría que hacer una interpretación extensiva de la configuración rawlsiana de la estructura básica para dar cuenta de que existen diferencias en las concepciones sobre expectativas y deseos individuales para obtener posiciones sociales u ocupar roles que obedecen a sistemas o estructuras de subordinación. Tales estructuras condicionan las posiciones de los peor situados y constituyen trabas sistémicas que impiden el desarrollo individual en libertad. Rawls (1995, 21) asume que sus principios de justicia puedan ser irrelevantes para las diversas convenciones y costumbres informales de la vida cotidiana, en las que, no obstante, se pueden integrar muchas de las complejas estructuras sociales que determinan en gran medida las posiciones de los individuos y sitúan a muchos sujetos en posiciones de ventaja o poder.

Es cierto que su escisión de la estructura básica en dos partes permite que los conflictos sociales y desequilibrios entre grupos se introduzcan en la ejecución de los principios igualitarios. No obstante, la 
prioridad que Rawls (1995, 492-493) otorga al primer principio de justicia hace que el respeto de cada persona a sí misma dependa esencialmente del estatus de igual ciudadanía, no de su posición en el orden social (Edmundson 2017, 114). En su reformulación de la justicia como equidad se acerca mucho a una idea de igualdad como no dominación y desigual estatus o posición. Entre las razones para regular las desigualdades señala las de "impedir que una parte de la sociedad domine al resto" y evitar un sistema de estatus, como bien posicional jerárquicamente organizado que se adscriba a condiciones como el nacimiento, el género o la raza (Rawls 2004, 177-178). Esta interpretación es, además, la más coherente con su concepción de la justicia como exigencia de estructuras adecuadas de justificación de las asimetrías sociales respecto de todos los implicados en un esquema cooperativo (Forst 2020, 157-158). Sin embargo, la solución que propone sigue disociando entre el plano político básico, abstracto e igualitario, de igual ciudadanía, y el plano de las desigualdades sociales y económicas reales que deben ser manejadas en la vida política ordinaria. Las políticas sociales son diseñadas una vez que los principios ideales han sido elegidos, de modo que estos pueden resultar ser un impedimento para atender las injusticias reales que interseccionan y crean formas complejas de dominación y subordinación que no quedan reflejadas en los esquemas abstractos (puede verse Mills 2005).

El debate sobre la dependencia de la teoría no ideal de una teoría ideal ha sido importante. Quienes critican la idealización de la teoría de la justicia rawlsiana consideran que es una guía insuficiente y errónea para abordar una pluralidad de formas de desventaja que asolan los contextos no ideales. Las idealizaciones terminan por distorsionar la ponderación adecuada de las diferentes demandas de justicia (Farrelly 2020, 70).

Rawls deja para la etapa legislativa el debate sobre las estrategias para la gestión de las desigualdades sociales y económicas. Superados el velo de la ignorancia y la etapa constituyente, entran en juego los conflictos entre intereses sociales y económicos diversos que se gestionan en sede legislativa. Reducir el principio de igualdad social a una aspiración política, que podría reflejarse en el preámbulo constitucional, evitaría el riesgo, en palabras de Rawls, de que los tribunales tuvieran que interpretar y hacer cumplir el principio de diferencia, para lo cual no están capacitados. Esto supone una limitación esencial al principio de di- 
ferencia que debería verse traducido en una serie de derechos que puedan exigirse al legislador y sean controlados jurisdiccionalmente (Moreso y Martí 2003).

\section{Las fronteras del principio de diferencia}

Más allá de las fronteras de una comunidad política encontramos algunas de las formas más brutales de desigualdad de nuestros días. No obstante, Rawls parte de una premisa que restringe el alcance de su propuesta igualitarista: sus principios de justicia están pensados para la estructura básica de la sociedad "concebida, por el momento, como un sistema cerrado, aislado de otras sociedades" (Rawls 1995, 21). No fue hasta 1993 cuando, por primera vez, se ocupó del problema de la justicia más allá de la frontera de un Estado. Sin embargo, algunas de las aportaciones más relevantes a una versión liberal igualitaria de una teoría de la justicia global se han construido sobre los cimientos de la teoría constructivista rawlsiana que exigen la mutua justificación de los principios aplicables. Su extensión al contexto global —sobre la base de la existencia de esquemas de interacción global con efectos profundos en la distribución de beneficios y cargas análogos a la estructura básica doméstica - representa una aproximación institucional a los problemas de desigualdad que cuestiona el orden vigente. Para autores como Charles Beitz (1979), Thomas Pogge (1994) o Allen Buchanan (2000), entre otros, la interdependencia global es una circunstancia básica de la justicia global que genera la necesidad de pensar en principios de justicia para un orden institucional compartido más allá de las fronteras.

Rawls no consideró, sin embargo, que los principios de justicia aplicables al ámbito estatal fueran también válidos para regir las desigualdades en una "sociedad de los pueblos" (Rawls 1999, 558). Los principios del Derecho de Gentes son más restringidos que los principios aplicables al ámbito estatal. Por una parte, no están orientados a la estructura básica internacional desde postulados individualistas, sino a regir la acción internacional de los pueblos. Por otra parte, no suponen un igualitarismo maximalista como el que impone la mejora de los más desaventajados. Su contenido se reduce a algunos derechos humanos esenciales y al deber de asistencia a los pueblos orientado a que cada uno sea miembro pleno y autónomo de la sociedad internacional. 
Las razones que Rawls proporciona para separar el modelo de justicia estatal del internacional no se han considerado suficientes. Por una parte, plantea el argumento de la autonomía política que descansa en una premisa implícita inaceptable relativa a que las causas de la desigualdad global radican en factores nacionales, que hacen que algunas sociedades prosperen y otras fracasen, obviando factores externos. Por otra parte, señala la falsa analogía entre individuos y pueblos como responsables de malas decisiones que producen consecuencias adversas, lo que supone limitar derechos individuales como consecuencia de decisiones erróneas de los gobernantes. Por último, el argumento de la falta de consenso global acerca de un modelo ideal de justicia distributiva, conforme al que no puede razonablemente esperarse la suficiente afinidad entre los pueblos acerca de requerimientos más exigentes, resulta inconsistente con las premisas individualistas de la construcción rawlsiana.

La propuesta de justicia de Rawls para el ámbito global ha recibido apoyos, sin embargo, desde las propias premisas de la justicia como equidad. Hugo Seleme (2011) destaca cómo no resulta incongruente con su concepción para el ámbito estatal, si se tiene en cuenta el carácter relacional o político de esta segunda. Es en el seno de comunidades políticas que se sustentan en lealtades institucionales y en compromisos recíprocos entre conciudadanos, en donde resulta más efectivo el mantenimiento de las instituciones, y la administración de los recursos humanos, materiales y culturales. Desde este enfoque político, no resulta incongruente la preferencia por un modelo internacional de justicia que refuerce las instituciones locales sobre un sistema que atienda directamente a la mejora de los peor situados a nivel global. Esto es así, si se considera que resulta más aceptable una propuesta de mejora de las instituciones internas de las sociedades más pobres, como pretende hacer el deber de asistencia, a sistemas de transferencia de recursos, en la medida en que puede esperarse que la primera mejore en mayor grado a los peor situados del planeta (Brock 2020).

Estos argumentos pretenden mostrar una mayor coherencia entre las dos teorías de la justicia de Rawls si se percibe su enfoque relacional o político. Pero apreciar dicha coherencia no es obstáculo para considerarla insuficiente con el compromiso de Rawls con los peor situados. Cabe una concepción alternativa de la justicia global desde sus propias premisas. Por una parte, no queda justificada la falta de disposición de 
Rawls a aceptar que el alcance del círculo de compromisos transnacionales es históricamente variable y que puede ampliarse a medida que las comunidades, las asociaciones y los individuos trabajen juntos en las instituciones cooperativas que desarrollen. Avanzar en ese sentido debe considerarse una obligación dinámica de justicia (Turégano 2016). Por otra parte, la base liberal igualitaria de la que parte Rawls requeriría tener en cuenta la posibilidad de que la realización de las concepciones del bien y los planes de vida individuales requiera el cruce de fronteras (Loewe 2007). El Derecho de Gentes asume que en el modelo ideal de sociedades liberales y decentes bien estructuradas desaparecería la inmigración como "problema serio" (Rawls 1999, 9). Pero la movilidad no es solo un problema, sino un elemento esencial en la definición de la idea de ciudadanía, si se concibe como un proyecto normativo abierto e inacabado (Turégano 2020).

\section{Bibliografía}

Arneson, R. 1999. Against Rawlsian Equality of Opportunity. Philosophical Studies 70, 77-112.

Beitz, C. 1979. Political Theory and International Relations. Princeton: Princeton University Press.

Brock, G. 2020. Rawls's Reasoning about International Economic Justice (329-342). En Mandle, J. y Roberts-Cady, S. (eds.), John Rawls. Oxford: Oxford University Press.

Buchanan, A. 2000. Rawls's Law of Peoples: Rules for a Vanished Westphalian World. Ethics 110, 697-721.

Cohen, G.A. 1997. Where the Action is: On the Site of Distributive Justice. Philosophy \& Public Affairs 26(1), 3-30.

Edmundson, W. 2017. John Rawls. Reticent Socialist. Cambridge: Cambridge University Press.

Farrelly, C. 2020. The 'Focusing Illusion' of Rawlsian Ideal Theory (61-72). En Mandle, J. y Roberts-Cady, S. (eds.), John Rawls. Oxford: Oxford University Press.

Forst, R. 2020. The Point of Justice (148-160). En Mandle, J. y Roberts-Cady, S. (eds.), John Rawls. Oxford: Oxford University Press.

Loewe, D. 2007. Inmigración y el derecho de gentes de John Rawls. Revista de Ciencia Política 27(2), 23-48.

Mills, C. 2005. 'Ideal Theory' as Ideology. Hypatia 20, 165-184.

Moreso, J.J. y Martí, J.L. 2003. La constitucionalización del principio de la diferencia. Revista de Ciencias Sociales 47, 545-574.

Piketty, T. 2019. Capital e ideología. Barcelona: Ediciones Deusto.

Pogge, T. 1994. An Egalitarian Law of Peoples. Philosophy and Public Affairs 23, 215219.

Pogge, T. 2007. Biography (3-27). En Pogge, T., John Rawls: His Life and Theory of Justice. Oxford: Oxford University Press. 
Rawls, J. 1995 [1971]. Teoría de la justicia. México DF: Fondo de Cultura Económica.

Rawls, J. 1999. The Law of Peoples. Cambridge, Mass: Harvard University Press.

Rawls, J. 2004 [2001]. La justicia como equidad. Una reformulación. Barcelona: Paidós.

Ribotta, S. 2021. El principio de diferencia y la aceptación de las desigualdades. Anales de la Cátedra Francisco Suárez 55, 281-305.

Seleme, H. 2011. Las fronteras de la justicia distributiva. Madrid: Centro de Estudios Políticos y Constitucionales.

Turégano, I. 2016. Distinguiendo los fundamentos de las responsabilidades globales (15-47). En Arcos, F. (ed.), La justicia y los derechos en un mundo globalizado. Madrid: Dykinson.

Turégano, I. 2020. Fronteras, movilidad y ciudadanía. Bajo Palabra 23, 131-162.

Vallespín, F. 2015. Cuando la riqueza campa a sus anchas. El País, 26 de enero. Disponible en: https://elpais.com/elpais/2015/01/26/opinion/1422286286_689929.html [11 de noviembre 2021].

Vallier, K. 2019. Rawls, Piketty, and the Critique of Welfare-State Capitalism. The Journal of Politics 81(1), 142-152. EP 